\title{
Short communication: Validation of somatic cell score-associated loci identified in a genome-wide association study in German Holstein cattle
}

\author{
Hamdy Abdel-Shafy, ${ }^{1}$ Ralf H. Bortfeldt, Monika Reissmann, and Gudrun A. Brockmann ${ }^{2}$ \\ Breeding Biology and Molecular Genetics, Department for Crop and Animal Sciences, Humboldt-Universität zu Berlin, Invalidenstraße 42, \\ 10115 Berlin, Germany
}

\begin{abstract}
Recently, we identified 6 genomic loci affecting daughter yield deviations (DYD) for somatic cell score (SCS) in a genome-wide association study (GWAS) performed with German Holstein bulls. In the current study, we tested if these loci were associated with SCS in cows using their own performance data. The study was performed with 1,412 German Holstein cows, of which 483 were daughters of 71 bulls that had been used in the GWAS. We tested 10 single nucleotide polymorphisms (SNP) representing 6 genomic regions that were associated with DYD for SCS in bulls. All tested SNP were significant in cows. Seven of them, located on Bos taurus autosomes (BTA) 6, 13, and 19, had the same direction of effect as those previously reported in the bull population. The most significant associations were detected on BTA6 and BTA19, accounting for $1.8 \%$ of the total genetic variance. The major allele of the 2 SNP on BTA6 and the minor allele of the 2 SNP on BTA19 were favorable for lower SCS. The differences between the homozygous genotype classes were up to 15,000 cells $/ \mathrm{mL}$. The verification of SNP associated with SCS in this study provides further evidence for the functional role of the linked genomic regions for immune response and contributes to identification of causative mutations. In particular, SNP with minor frequency of the favorable allele possess high potential to reduce SCS in German Holstein cattle by selection. Key words: validation study, single nucleotide polymorphism, mastitis resistance, candidate gene
\end{abstract}

\section{Short Communication}

Beside management strategies, genetic improvement is an important tool to control mastitis in dairy farms. Because clinical mastitis and its indicator SCS have low

Received June 17, 2013.

Accepted December 16, 2013.

${ }^{1}$ Present address: Department of Animal Production, Faculty of Agriculture, Cairo University, 6 El-Gamma street, 12613 Giza, Egypt.

${ }^{2}$ Corresponding author: gudrun.brockmann@agrar.hu-berlin.de heritabilities of about 0.10 and 0.16 in German Holstein cattle, respectively, traditional methods of selection have limited success (Martin et al., 2010; Hinrichs et al., 2011). Alternatively, selection based on genomic information is expected to be more accurate, to cost less, and to become sustainable practice (Meuwissen et al., 2001). However, because most SNP used for genomic selections are linked to unknown causative mutations, linkage disequilibrium (LD) must be adjusted from time to time because of recombination events. To circumvent re-evaluation of SNP effects and to understand the biological mechanism of gene variants, it is desirable to know the causative mutations. An essential step is the accurate genomic mapping of genes contributing to SCS and clinical mastitis.

Previously, we identified 16 SNP on 6 chromosomal regions that were significantly associated with daughter yield deviations (DYD) of SCS in a genome-wide association study (GWAS) in German Holstein bulls (H. Abdel-Shafy, R. H. Bortfeldt, J. Tetens, and G. A. Brockmann, unpublished data). The significant SNP were located on BTA 5, 6, 13, 18, and 19, and on the $\mathrm{X}$ chromosome. Among these associated loci, the chromosomal region on BTA6 between 85.5 and 88.1 $\mathrm{Mb}$ (position is based on UMD3.1 assembly; http:// www.ensembl.org) was also significant in US Holstein cattle (Cole et al., 2011), which provides evidence for the importance of this locus in the regulation of SCS in dairy cattle.

Although GWAS are powerful to identify genomic loci with high precision, false-positive associations due to confounding effects are considered a limiting factor. The most important effect regarding this issue is population stratification (Goddard and Hayes, 2009). However, even after correction for population stratification, some false results might still remain. Therefore, validation studies are important to test whether the $\mathrm{LD}$ between a causative mutation and a SNP is the same between populations and is not affected by the population sub-structure (Chanock et al., 2007). For validation, animals with recently recorded data that were not used in the initial discovery study - for example, cows - can be used. In contrast to DYD of bulls, performance data 
of cows allows us to test additive and dominance effects (Lush, 1935). Therefore, the objectives of this study were to validate associations obtained from a GWAS for DYD of SCS in bulls (H. Abdel-Shafy, R. H. Bortfeldt, J. Tetens, and G. A. Brockmann, unpublished data) in a cow population and to test the mode of inheritance of associated loci.

The study was carried out with 1,412 German Holstein cows descending from 284 sires; 71 of these bulls were used in the initial GWAS, in which we detected significant SNP for DYD of SCS (H. Abdel-Shafy, R. H. Bortfeldt, J. Tetens, and G. A. Brockmann, unpublished data). The average number of cows per sire was 5 with a minimum of 1 (122 cases) to a maximum of 80 (1 case). The cows were born between 1996 and 2005 and calved between 1999 and 2010, with more than 87\% calving after 2003. The age at first calving for these cows ranged from 620 to $1,250 \mathrm{~d}$, with an average of 840 d. The cows were kept on 3 dairy farms in northern Germany under similar management conditions. Records from monthly test-days of SCC were provided by the center of national breeding evaluation (VIT: Vereinigte Informationssysteme Tierhaltung, Verden, Germany). To ensure a homogeneous data set, only the first 3 lactations were used. Furthermore, records between 5 and 305 DIM and lactations with a minimum of 3 testdays were accepted. Using these criteria, the data set included 1,412 cows with 36,813 records. Among these cows, 1,401, 1,370, and 1,156 animals finished the first, second, and third lactations, respectively. The SCC in the final data set was transformed using a logarithmic transformation function (Ali and Shook, 1980) to obtain a distribution (SCS) that is closer to normal: SCS $=\log _{2}(\mathrm{SCC} / 100)+3$.

Genomic DNA was extracted from lymphocytes using the NucleoSpin Blood Quick Pure Kit (MachereyNagel, Düren, Germany). Cows were genotyped for 10 SNP in 6 regions on BTA5, 6, 13, 18, 19, and chromosome X (Table 1), which were associated with DYD for SCS in our previous GWAS with bulls (H. Abdel-Shafy, R. H. Bortfeldt, J. Tetens, and G. A. Brockmann, unpublished data). Genotyping was performed with allelespecific primers (Table 1) in PCR assays as described previously (Kreuzer et al., 2013); the genotyping rate was $98.6 \%$.

To test the association between single SNP and SCS, a mixed model was applied using the MIXED procedure of SAS (version 9.3 SAS Institute Inc., Cary, NC). The model considered the lactation curve fit of Ali and Schaeffer (1987). Test-day records within lactation of a cow were treated as repeated measurements. The Schwarz Bayesian information criterion was used to select an appropriate covariance structure in the re- peated statement (Schwarz, 1978). Because of unequal distances between test-days, the spatial power covariance structure was used as an appropriate fit for the data set. The final model was

$$
\begin{gathered}
\mathrm{Y}_{\mathrm{ijklmnop}}=\mu+\mathrm{G}_{\mathrm{i}}+\mathrm{AC}_{\mathrm{j}}+\mathrm{b}_{\mathrm{k} 1}(\mathrm{DIM} / \mathrm{c}) \\
+\mathrm{b}_{\mathrm{k} 2}(\mathrm{DIM} / \mathrm{c})^{2}+\mathrm{b}_{\mathrm{k} 3} \log (\mathrm{c} / \mathrm{DIM})+\mathrm{b}_{\mathrm{k} 4}[\log (\mathrm{c} / \mathrm{DIM})]^{2} \\
+\mathrm{b}_{1}(\mathrm{MY})+\mathrm{p}_{\mathrm{m}}+\text { hys }_{\mathrm{n}}+\mathrm{s}_{\mathrm{o}}+\varepsilon_{\mathrm{ijklmnop}},
\end{gathered}
$$

where $Y_{\mathrm{ijklmnop}}$ is the test-day record of SCS, $\mu$ is the overall mean of observations, $G_{i}$ is the fixed effect of the SNP genotypes, $\mathrm{AC}_{\mathrm{j}}$ is the fixed effect of age at calving, $b_{k 1}$ to $b_{k 4}$ are the regression coefficients associated with the fixed lactation function, where DIM is days in milk and c is a constant set to 305 (Ali and Schaeffer, 1987), and $b_{1}$ is the regression coefficient associated with the fixed effect of milk yield (MY; Jamrozik and Schaeffer, 2010). Whereas the random environmental effect between consecutive test-day records within each lactation of a cow was included in the term of $\mathrm{p}_{\mathrm{m}}$, the environmental differences between consecutive lactations were considered as a random effect in the term of hys $s_{n}$ [hys is a combined effect of herd $(\mathrm{h})$, year $(\mathrm{y})$, and season (s) of calving, where seasons were defined as calendar quarters: January to March, April to June, July to September, and October to December]. We accounted for population stratification by fixing the sire $\mathrm{s}_{\mathrm{O}}$ as a random effect, and $\varepsilon_{\mathrm{ijk} k \mathrm{mnop}}$ is the residual error.

Single nucleotide polymorphisms were considered significant at a threshold of $\alpha \leq 0.05$ after Bonferroni correction if the nominal $P$-value $\times 10 \leq 0.05$, where 10 is the number of tested SNP. Post hoc, effects among genotype classes were tested for significance using a Tukey-Kramer test as implemented in SAS (SAS Institute Inc.). In addition, additive and dominance effects were tested. For estimation of the genetic variance explained by significant SNP, we used the formula $2 \beta^{2} f$ $(1-f)$, where $\beta$ denotes the regression coefficient; that is, the effect of the locus per copy of the variant, and $f$ denotes the frequency of the variant (Park et al., 2010). We used $5^{\prime}$ and $3^{\prime}$ flanking regions of $1 \mathrm{Mb}$ around significant SNP to search for candidate genes that could be linked to the significant SNP. Gene search was performed via the Ensembl database (UMD3.1 Ensembl database build 73; http://www.ensembl.org).

The minor allele frequencies of all tested SNP ranged from 0.14 to 0.41 in the cow population, which were almost the same as in the bull population (ranging from 0.14 to 0.43 ; Table 2). All 10 tested SNP were significantly associated with SCS after Bonferroni correction $(P \leq 0.005)$. These SNP were located on BTA5, 
Table 1. Single nucleotide polymorphisms genotyped in the study ${ }^{1}$

\begin{tabular}{|c|c|c|c|c|}
\hline SNP ID & Chromosome & Position (bp) & Primer & Primer sequence \\
\hline \multirow[t]{3}{*}{ rs41257360 } & 5 & 97948752 & A1 & GAAGGTGACCAAGTTCATGCTCTCCAGATCAATCTTGAGTAGT \\
\hline & & & A2 & GAAGGTCGGAGTCAACGGATTCTCTCCAGATCAATCTTGAGTAGC \\
\hline & & & Common primer & CTCAGACGTCTGGATCCTCGGAT \\
\hline \multirow[t]{3}{*}{ rs41588957 } & 6 & 85527109 & A1 & GAAGGTGACCAAGTTCATGCTAGAACTCTCATTCAGTTCAGTCCC \\
\hline & & & $\mathrm{A} 2$ & GAAGGTCGGAGTCAACGGATTCAGAACTCTCATTCAGTTCAGTCCT \\
\hline & & & Common primer & GTTCTGCGATTCATGGGGTCACAAA \\
\hline \multirow[t]{3}{*}{ rs 110707460} & 6 & 88069548 & A1 & GAAGGTGACCAAGTTCATGCTCCCAGTTCTAGGCCCTGGAAAT \\
\hline & & & A2 & GAAGGTCGGAGTCAACGGATTCCAGTTCTAGGCCCTGGAAAC \\
\hline & & & Common primer & TGGAATATAGGTTTCATGAGTTGGGACTT \\
\hline \multirow[t]{3}{*}{ rs109934030 } & 13 & 78644697 & A1 & GAAGGTGACCAAGTTCATGCTGATGCCACATCTACCGAGTTACG \\
\hline & & & A2 & GAAGGTCGGAGTCAACGGATTGATGCCACATCTACCGAGTTACA \\
\hline & & & Common primer & GCACAAGTTTGGTCTTCAATGTCATCTTA \\
\hline \multirow[t]{3}{*}{ rs41634110 } & 13 & 79730805 & A1 & GAAGGTGACCAAGTTCATGCTAGCACACCTGTAGCAAAGAGAAACT \\
\hline & & & A2 & GAAGGTCGGAGTCAACGGATTCACACCTGTAGCAAAGAGAAACC \\
\hline & & & Common primer & GGTGACCACCTGTGCACTTTAGTTT \\
\hline \multirow[t]{3}{*}{ rs109441194 } & 13 & 80094921 & A1 & GAAGGTGACCAAGTTCATGCTAGTGCATGGGGTAAGTATTGTTCG \\
\hline & & & A2 & GAAGGTCGGAGTCAACGGATTGAGTGCATGGGGTAAGTATTGTTCA \\
\hline & & & Common primer & CACACCATCATACTCACTGGCAACTA \\
\hline \multirow[t]{3}{*}{ rs29020544 } & 18 & 43327273 & A1 & GAAGGTGACCAAGTTCATGCTGGGGCATATTGTGACCCAGTG \\
\hline & & & A2 & GAAGGTCGGAGTCAACGGATTCTGGGGCATATTGTGACCCAGTT \\
\hline & & & Common primer & GAATATATTTAGGGCTTGGTTTTTAGGGAT \\
\hline \multirow[t]{3}{*}{ rs41257403 } & 19 & 50647677 & A1 & GAAGGTGACCAAGTTCATGCTCGCTCAGACAGTTGAGCTCCTT \\
\hline & & & A2 & GAAGGTCGGAGTCAACGGATTGCTCAGACAGTTGAGCTCCTC \\
\hline & & & Common primer & GGACGATGTGGGGGTGATGCAT \\
\hline \multirow[t]{3}{*}{ rs41636878 } & 19 & 52436005 & A1 & GAAGGTGACCAAGTTCATGCTTTAAAAATTAAGAACAAATAACCCCCTC \\
\hline & & & & GAAGGTCGGAGTCAACGGATTGCTTTAAAAATTAAGAACAAATAACCCCCTT \\
\hline & & & Common primer & GGTGTATTTGCTCTGTGCCTGCTT \\
\hline \multirow[t]{3}{*}{ rs41629005 } & $\mathrm{X}$ & 30639394 & A1 & GAAGGTGACCAAGTTCATGCTGTATTTATTAGAGCAAATTAGCGTCTGTC \\
\hline & & & & GAAGGTCGGAGTCAACGGATTGTATTTATTAGAGCAAATTAGCGTCTGTT \\
\hline & & & Common primer & ATGAAGCCATGTCTCTGATCATCCATTT \\
\hline \multicolumn{5}{|c|}{$\begin{array}{l}{ }_{1}^{1} \text { Primers A1 and A2 are primers for the alternative alleles } 1 \text { and 2, respectively. Positions are given according to the University of Maryland bovine genome assembly (UMD3.1; } \\
\text { http://www.ensembl.org). }\end{array}$} \\
\hline
\end{tabular}


$6,13,18,19$, and the $\mathrm{X}$ chromosome, with an effect size between $-0.001(12,510$ cells $/ \mathrm{mL})$ and $0.13(13,700$ cells $/ \mathrm{mL}$ ) units of SCS (Table 2).

Seven significantly associated SNP had same direction of effect as those originally reported in the bull study. The same direction of effect of these SNP was further verified by the high correlation (0.61) between the changes per minor allele $(\beta)$ in both populations. The other 3 SNP were also significant but had effects in the opposite direction in the cow population (Table 2 ). The opposite direction of effect might be due to weak LD between the tested SNP and the causative mutation. In this case, the effect of the tested SNP strongly depends on the analyzed population. The opposite direction of allele effect suggests linkage to hidden mutations that distort the observed genotypic effect found in another population. In addition, interaction between genomic loci can modify the classical modes of inheritance, including the direction of effect.

The most significant SNP $(P<0.0001)$ in the cow population were $r s 41588957$ and $r s 110707460$ on BTA6. The major alleles of these 2 SNP were associated with lower SCS. The difference between the homozygous genotype classes for both associated SNP was 15,000 cells $/ \mathrm{mL}$ (Figure 1). In contrast, the minor alleles of the highly significant markers $(P<0.0001)$ on BTA19 (rs41257403 and rs41636878) were associated with lower SCS (Table 2).

The SNP on BTA6 and BTA19, which were verified in the cow population, are located in QTL that were repeatedly mapped for mastitis traits in German, French, and Danish Holstein cattle by linkage analysis in structured pedigrees (Bennewitz et al., 2004; Lund et al., 2008). The validated SNP on BTA13 have not been reported before in either Holstein or other cattle breeds (http://www.animalgenome.org/cgi-bin/QTLdb/BT/ index). Compared with the confidence interval from previously reported QTL in structured pedigrees, this confirmation study together with the GWAS in bulls contributes effectively to shorten the genomic regions where the underlying genes are located. For instance, the QTL for SCS on BTA6 with a peak QTL position at $99 \mathrm{cM}$ and a confidence interval of $119 \mathrm{cM}$ in the German Holstein population (Bennewitz et al., 2004) could be fine-mapped in our study to a region of about $4.54 \mathrm{Mb}$, comprising the interval between 85.53 and 88.07 , where the 2 significant loci reside, and a $1-\mathrm{Mb}$ flanking region on both sides. Likewise, the QTL previously identified on BTA19 for SCS with a peak QTL position at $58 \mathrm{cM}$ and a confidence interval of $123 \mathrm{cM}$ (Bennewitz et al., 2004) could be reduced to a region of about $3.79 \mathrm{Mb}$ linked to the interval between 50.65 and $52.44 \mathrm{Mb}$, where the 2 significant $\mathrm{SNP} \pm 1 \mathrm{Mb}$ flanking region reside.

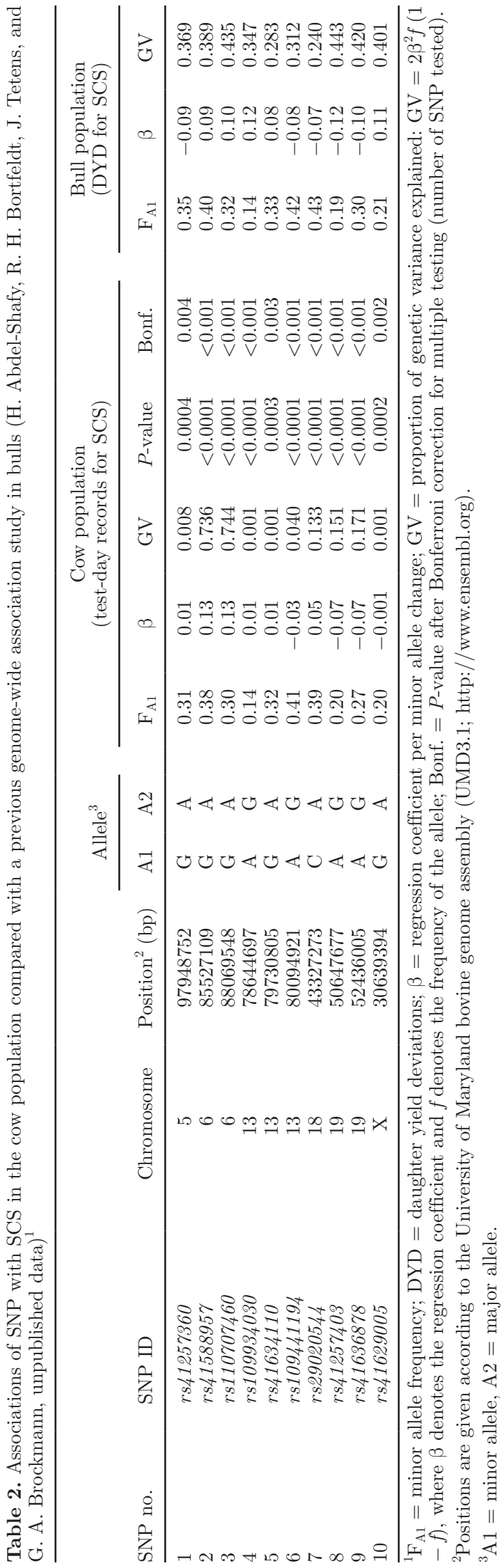


A

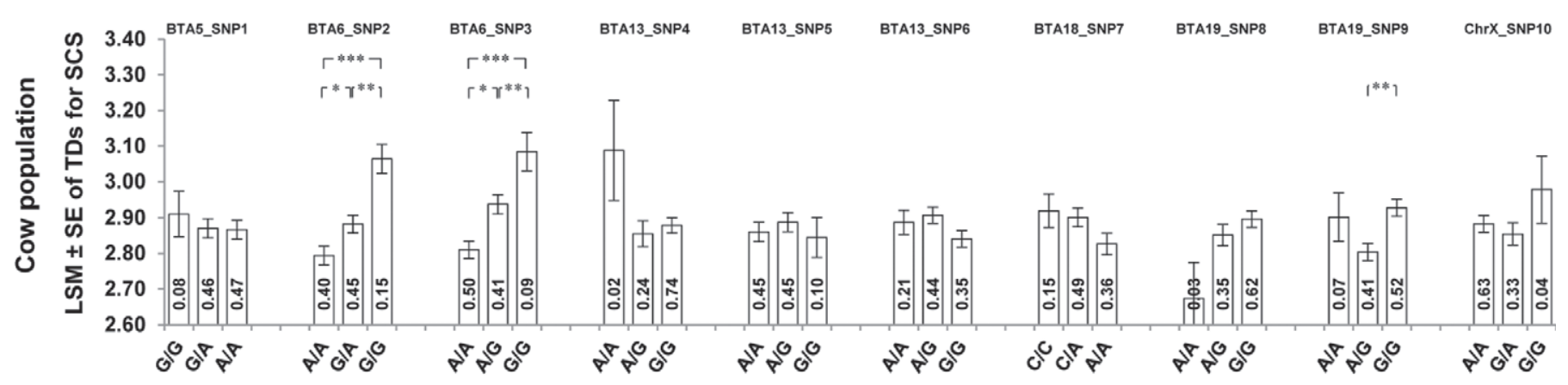

B

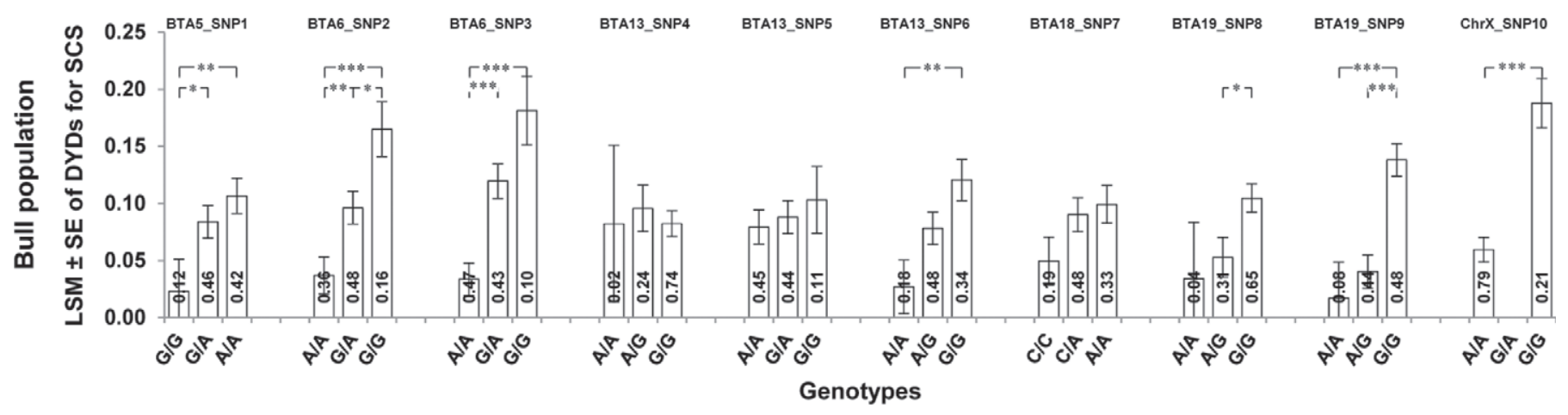

Figure 1. Genotype effect plots of SNP associated with SCS in the (A) cow population and (B) bull population (H. Abdel-Shafy, R. H. Bortfeldt, J. Tetens, and G. A. Brockmann, unpublished data). DYD = daughter yield deviations, TD = test-day records, Chr $=$ chromosome, $\mathrm{SNP} 1=r s 41257360, \mathrm{SNP} 2=r s 41588957, \mathrm{SNP} 3=r s 110707460, \mathrm{SNP} 4=r s 109934030, \mathrm{SNP} 5=r s 41634110, \mathrm{SNP} 6=r s 109441194, \mathrm{SNP} 7=$ rs29020544, SNP8 $=$ rs41257403, SNP9 $=$ rs41636878, and SNP10 $=$ rs41629005. ${ }^{*} P<0.05, * * P<0.01$, and ${ }^{* * *} P<0.001$ indicate significant differences between groups. Numbers inside the bars indicate genotype frequency.

The significant region on BTA6 in our study coincided very well with the results of a GWAS performed in American Holstein cattle for SCS, which identified 3 significant SNP between 85 and $89 \mathrm{Mb}$ on BTA6 (Cole et al., 2011). Although the same BovineSNP50 BeadChip (Illumina Inc., San Diego, CA) was used in both studies (Cole et al., 2011; H. Abdel-Shafy, R. H. Bortfeldt, J. Tetens, and G. A. Brockmann, unpublished data), different SNP were significant in both studies. This is likely due to the different LD phases between SNP and the unknown causative mutation in both populations. The mapping of the same region on BTA6 in 2 independent populations of Holstein cattle with different experimental and analytical setups increases the confidence that the linked neighborhood region contains true causative mutations affecting SCS.

With respect to the mode of inheritance, the alleles of the 2 SNP on BTA6 and 1 SNP on BTA19 indicated an additive mode of inheritance (Figure 1). Although the SNP on BTA5, 13, 18, and chromosome X showed no significant differences between genotype classes in pairwise tests, the mode of inheritance for those SNP seems to be dominance.
The effect size of the associated SNP was smaller in cows than in bulls. The total genetic variance explained by the 7 confirmed SNP was $1.84 \%$ in the cow population, whereas it was $2.63 \%$ in the bull population for the same SNP. Even though the effects are small, they seem to be consistent in both populations (Table 2).

Markers associated with SCS are located close to several promising candidate genes. For example, the SNP rs110707460 on BTA6 is located within the deoxycytidine kinase gene $(D C K)$, which has a functional role in drug resistance and sensitivity (van den Heuvel-Eibrink et al., 2001). The SNP rs109441194 on BTA13 is located in the gene encoding the cytoplasmic nuclear factor of activated T-cell calcineurin-dependent 2 fragment (NFATC2), which plays a central role in the transcriptional activation of T-cell cytokine genes during the immune response, especially IL-2, IL-3, IL-4, tumor necrosis factor- $\alpha$, and granulocyte-macrophage colony-stimulating factor (Rao, 1994). The position of SNP rs41257403 on BTA19 is $2.67 \mathrm{~kb}$ away from the forkhead box protein K2 (FOXK2) gene, which is necessary for the transcriptional regulation of IL-2 (Li et al., 1991). 
In conclusion, this association analysis in cows confirmed 7 out of 10 SNP previously identified for DYD of SCS in bulls - a high accordance rate. Although this accordance is due, in part, to the same base population of German Holstein cattle, it mirrors the good model fit with respect to population stratification, which was considered by different approaches in the bull and cow studies. The validation study in cows provides evidence for the importance of loci on BTA6, 13, and 19 for SCS as an indication for mastitis susceptibility. Because favorable alleles segregate with minor frequencies on BTA19, the potential exists to reduce SCS by genomic selection. The further identification of the causative mutations would be desirable to allow direct selection for the beneficial allele without the disadvantage of using linked markers, as is presently done with genomic selection.

\section{ACKNOWLEDGMENTS}

This study was supported by the Bundesministerium für Bildung und Forschung (BMBF) as a part of the GenoTrack project in the Network "Funktionelle GenomAnalyse im Tierischen Organismus (FUGATO; Ref. No: 0315 134B). We gratefully thank Uwe Müller (Humboldt-Universität, Berlin, Germany) for data preparation, and Birgit Rudolphi (Landesforschungsanstalt für Landwirtschaft und Fischerei, Dummerstorf, Germany), Uwe Brockmann (Agricon GmbH, Rostock, Germany), and three farms for providing the cow data. Hamdy Abdel-Shafy acknowleges support from the Deutscher Akademischer Austauschdienst (DAAD, Bonn, Germany) and the Ministry of Higher Education and Scientific Research of the Arab Republic of Egypt (MHESR, Cairo, Egypt).

\section{REFERENCES}

Ali, A. K. A., and G. E. Shook. 1980. An optimum transformation for somatic cell concentration in milk. J. Dairy Sci. 63:487-490.

Ali, T. E., and L. R. Schaeffer. 1987. Accounting for covariances among test-day milk yields in dairy cows. Can. J. Anim. Sci. 67:637-644.

Bennewitz, J., N. Reinsch, V. Guiard, S. Fritz, H. Thomsen, C. Looft, C. Kuhn, M. Schwerin, C. Weimann, G. Erhardt, F. Reinhardt, R. Reents, D. Boichard, and E. Kalm. 2004. Multiple quantitative trait loci mapping with cofactors and application of alternative variants of the false discovery rate in an enlarged granddaughter design. Genetics 168:1019-1027.
Chanock, S. J., T. Manolio, M. Boehnke, E. Boerwinkle, D. J. Hunter, G. Thomas, J. N. Hirschhorn, G. Abecasis, D. Altshuler, J. E. Bailey-Wilson, L. D. Brooks, L. R. Cardon, M. Daly, P. Donnelly, J. F. Fraumeni Jr., N. B. Freimer, D. S. Gerhard, C. Gunter, A. E. Guttmacher, M. S. Guyer, E. L. Harris, J. Hoh, R. Hoover, C. A. Kong, K. R. Merikangas, C. C. Morton, L. J. Palmer, E. G Phimister, J. P. Rice, J. Roberts, C. Rotimi, M. A. Tucker, K. J. Vogan, S. Wacholder, E. M. Wijsman, D. M. Winn, and F. S. Collins. 2007. Replicating genotype-phenotype associations. Nature 447:655-660.

Cole, J. B., G. R. Wiggans, L. Ma, T. S. Sonstegard, T. J. Lawlor Jr., B. A. Crooker, C. P. Van Tassell, J. Yang, S. Wang, L. K. Matukumalli, and Y. Da. 2011. Genome-wide association analysis of thirty one production, health, reproduction and body conformation traits in contemporary U.S. Holstein cows. BMC Genomics $12: 408$.

Goddard, M. E., and B. J. Hayes. 2009. Mapping genes for complex traits in domestic animals and their use in breeding programmes. Nat. Rev. Genet. 10:381-391.

Hinrichs, D., J. Bennewitz, E. Stamer, W. Junge, E. Kalm, and G. Thaller. 2011. Genetic analysis of mastitis data with different models. J. Dairy Sci. 94:471-478.

Jamrozik, J., and L. R. Schaeffer. 2010. Recursive relationships between milk yield and somatic cell score of Canadian Holsteins from finite mixture random regression models. J. Dairy Sci. 93:54745486

Kreuzer, S., M. Reissmann, and G. A. Brockmann. 2013. Gene test to elucidate the ETEC F4ab/F4ac receptor status in pigs. Vet. Microbiol. 162:293-295.

Li, C., C. F. Lai, D. S. Sigman, and R. B. Gaynor. 1991. Cloning of a cellular factor, interleukin binding factor, that binds to NFAT-like motifs in the human immunodeficiency virus long terminal repeat. Proc. Natl. Acad. Sci. USA 88:7739-7743.

Lund, M. S., B. Guldbrandtsen, A. J. Buitenhuis, B. Thomsen, and C. Bendixen. 2008. Detection of quantitative trait loci in Danish Holstein cattle affecting clinical mastitis, somatic cell score, udder conformation traits, and assessment of associated effects on milk yield. J. Dairy Sci. 91:4028-4036.

Lush, J. L. 1935. Progeny test and individual performance as indicators of an animal's breeding value. J. Dairy Sci. 18:1-19.

Martin, G., R. Schafberg, and H. H. Swalve. 2010. Udder health data in dairy cattle breeding: An alternative approach for genetic evaluation. Pages 1-18 in 9th World Congr. Genet. Appl. Livest. Prod. (WCGALP), Leipzig, Germany. Event lab GmbH, Leipzig, Germany.

Meuwissen, T. H. E., B. J. Hayes, and M. E. Goddard. 2001. Prediction of total genetic value using genome-wide dense marker maps. Genetics 157:1819-1829.

Park, J. H., S. Wacholder, M. H. Gail, U. Peters, K. B. Jacobs, S. J. Chanock, and N. Chatterjee. 2010. Estimation of effect size distribution from genome-wide association studies and implications for future discoveries. Nat. Genet. 42:570-575.

Rao, A. 1994. NF-ATp: A transcription factor required for the coordinate induction of several cytokine genes. Immunol. Today $15: 274-281$.

Schwarz, G. 1978. Estimating the dimension of a model. Ann. Stat. 6:461-464.

van den Heuvel-Eibrink, M. M., E. A. Wiemer, M. Kuijpers, R. Pieters, and P. Sonneveld. 2001. Absence of mutations in the deoxycytidine kinase $(d C K)$ gene in patients with relapsed and/or refractory acute myeloid leukemia (AML). Leukemia 15:855-856. 